\title{
Medial knee joint contact force in the intact limb during walking in recently ambulatory service members with unilateral limb loss: cross-sectional study
}

\author{
Ross H Miller ${ }^{\text {Corresp., }}{ }^{1,2}$ ， Rebecca L Krupenevich ${ }^{1}$ ， Alison L Pruziner ${ }^{1,3,4}$, Erik J Wolf ${ }^{3,4}$ ， Barri L Schnall ${ }^{3}$ \\ 1 Department of Kinesiology, University of Maryland, College Park, Maryland, United States \\ Neuroscience \& Cognitive Science Program, University of Maryland, College Park, Maryland, United States \\ 3 Walter Reed National Military Medical Center, Bethesda, Maryland, United States \\ 4 DoD-VA Extremity Trauma and Amputation Center of Excellence, United States \\ Corresponding Author: Ross H Miller \\ Email address: rosshm@umd.edu
}

Background: Individuals with unilateral lower limb amputation have a high risk of developing knee osteoarthritis (OA) in their intact limb as they age. This risk may be related to joint loading experienced earlier in life. We hypothesized that loading during walking would be greater in the intact limb of young US military Service Members with limb loss than in controls with no limb loss.

Methods: cross-sectional instrumented gait analysis at self-selected walking speeds with a limb loss group ( $N=10$, age $27 \pm 5$ years, $170 \pm 36$ days since last surgery) including five service members with transtibial limb loss and five with transfemoral limb loss, all walking independently with their first prosthesis for approximately two months. Controls ( $\mathrm{N}=10$, age $30 \pm 4$ years) were service members with no overt demographical risk factors for knee OA. 3D inverse dynamics modeling was performed to calculate joint moments and medial knee joint contact forces (JCF) were calculated using a reductionbased musculoskeletal modeling method and expressed relative to body weight (BW).

Results: Peak JCF and maximum JCF loading rate were significantly greater in limb loss (184 \% BW, 2469 $\% \mathrm{BW} / \mathrm{s}$ ) vs. controls (157 \%BW, $1985 \% \mathrm{BW} / \mathrm{s}$ ), with large effect sizes. Results were robust to probabilistic perturbations to the knee model parameters.

Discussion: Assuming these data are reflective of joint loading experienced in daily life, they support a "mechanical overloading" hypothesis for the risk of developing knee OA in the intact limb of limb loss subjects. Examination of the evolution of gait mechanics, joint loading, and joint health over time, as well as interventions to reduce load or strengthen the ability of the joint to withstand loads, is warranted. 
1 Title:

2 Medial knee joint contact force in the intact limb during walking in recently ambulatory service

3 members with unilateral limb loss: cross-sectional study

4

5 Authors:

6 Ross H. Miller ${ }^{1,2}$ (corresponding author)

7 Rebecca L. Krupenevich ${ }^{1}$

8 Alison L. Pruziner ${ }^{1,3,4}$

9 Erik J. Wolf ${ }^{3,4}$

10 Barri L. Schnall ${ }^{4}$

11

$12{ }^{1}$ Department of Kinesiology, University of Maryland, College Park, MD, USA

13 2Neuroscience \& Cognitive Science Program, University of Maryland, College Park, MD, USA

$14{ }^{3}$ DoD-VA Extremity Trauma and Amputation Center of Excellence

$15{ }^{4}$ Walter Reed National Military Medical Center, Bethesda, MD, USA

Corresponding author:

18 Ross H. Miller, rosshm@umd.edu 
19 ABSTRACT

20 Background: Individuals with unilateral lower limb amputation have a high risk of developing 21 knee osteoarthritis (OA) in their intact limb as they age. This risk may be related to joint loading 22 experienced earlier in life. We hypothesized that loading during walking would be greater in the 23 intact limb of young US military Service Members with limb loss than in controls with no limb 24 loss.

Methods: cross-sectional instrumented gait analysis at self-selected walking speeds with a limb loss group ( $N=10$, age $27 \pm 5$ years, $170 \pm 36$ days since last surgery) including five service members with transtibial limb loss and five with transfemoral limb loss, all walking independently with their first prosthesis for approximately two months. Controls $(\mathrm{N}=10$, age $30 \pm 4$ years) were service members with no overt demographical risk factors for knee OA. 3D inverse dynamics modeling was performed to calculate joint moments and medial knee joint contact forces (JCF) were calculated using a reduction-based musculoskeletal modeling method and expressed relative to body weight (BW).

Results: Peak JCF and maximum JCF loading rate were significantly greater in limb loss (184 \% BW, $2469 \% \mathrm{BW} / \mathrm{s})$ vs. controls $(157 \% \mathrm{BW}, 1985 \% \mathrm{BW} / \mathrm{s})$, with large effect sizes. Results were robust to probabilistic perturbations to the knee model parameters.

Discussion: Assuming these data are reflective of joint loading experienced in daily life, they support a "mechanical overloading" hypothesis for the risk of developing knee OA in the intact limb of limb loss subjects. Examination of the evolution of gait mechanics, joint loading, and joint health over time, as well as interventions to reduce load or strengthen the ability of the joint 43 to withstand loads, is warranted. 


\section{INTRODUCTION}

45

46

47

48

49

50

51

52

53

54 55 56

57

Since 2001, over 1,600 United States military service members have sustained traumatic injuries involving major limb loss (Fischer, 2015). Individuals with unilateral lower limb loss have a high risk of developing secondary physical conditions later in life, including osteoarthritis (OA) in their intact limb (Gailey et al., 2008; Morgenroth et al., 2012). In veterans with unilateral limb loss, the prevalence of knee OA is $30-90 \%$ greater in the intact limb compared to veterans without limb loss (Hungerford \& Cockin, 1975; Lemaire \& Fisher, 1994; Norvell et al., 2005). Most of the subjects in these previous studies were older adults who had been living with their amputations for several decades. Recent reviews have indicated a rising incidence of idiopathic knee OA in the young military population (Showery et al., 2016) and in service members with limb loss specifically (Farrokhi et al., 2016). The younger service members with limb loss from recent conflicts may therefore live with a relatively high risk of developing knee OA for many years.

Our long-term goal is to develop interventions that can be implemented early after limb loss to minimize the risk of developing knee OA later in life. Achieving this goal is challenging because the causal mechanisms of OA are unknown. However, mechanical loading is suspected to play a major role in the disease's etiology (Andriacchi \& Mündermann, 2006; Maly, 2008;

Felson, 2013), and overloading the intact limb by deliberately or subconsciously favoring it during activities of daily living is a long-standing hypothesis for explaining the prevalence of knee OA in the limb loss population (Borgmann, 1960). The fatigue life of human articular cartilage in vitro suggests that the stresses from repetitive loading in walking could produce mechanical failure of the superficial collagen fibers well within the human lifespan (Weightman et al., 1978; Bellucci \& Seedhom, 2001). Relatedly, the "cartilage conditioning” hypothesis 
67 argues that cartilage in vivo adapts to withstand frequently encountered stress levels (Seedhom,

68 2006). If abrupt changes in gait mechanics due to amputation and prosthesis use result in sudden

69 increases in loading of the intact limb, these loads could overwhelm the adaptive response of

70 cartilage, particularly if it has recently been weakened due to a long period of unloading from

71 injury, surgery, and recovery. For example, knee cartilage glycosaminoglycan content, which

72 affects the compressive stiffness of cartilage, remains below baseline for at least a year following

73 six weeks of immobilization in humans (Owman et al., 2014). Similar results are seen in animal

74 models (Jurvelin et al., 1986). The time when unilateral limb loss patients first begin walking

75 again could therefore be a particularly important time to assess their joint loading.

In this study, we therefore examined knee joint loading in the intact limb of relatively

77 young service members with unilateral limb loss who had recently begun walking independently

78 with their prostheses for the first time. We tested the hypothesis that loading of the medial knee

79 joint, as indicated by the peak, loading rate, and impulse of the medial joint contact force, is

80 greater during self-paced walking in the intact limb of young service members with limb loss

81 than in a control group of similar age and background (young service members) without limb

82 loss. These three outcome variables were chosen because they have all previously been

83 associated with knee OA risk, and it is unknown which is most important. The medial knee was

84 chosen because the knee is the most common site of OA (Centers for Disease Control and

85 Prevention, 2015), and because medial knee OA is more common than lateral knee OA (Wise et

86 al., 2012). An elevated risk of general knee OA has been reported in young military service

87 members with limb loss (Farrokhi et al., 2016). The risk of medial knee OA specifically in this

88 population is unknown, but bone mineral density and joint structure suggest a high risk for

89 medial knee OA in this population (Royer \& Koenig, 2005; Morgenroth et al., 2014). 
Recent studies suggest that the peak external knee adduction moment (KAM), the most widely-used metric for quantifying medial knee joint loading in gait (Foroughi et al., 2009; Simic et al., 2011), is similar in the intact limb of young service members with limb loss vs. controls

93 (Pruziner et al., 2014; Esposito \& Wilken, 2014), and that including the external knee flexion 94 moment (KFM) more accurately estimates medial joint loading than using the KAM alone 95 (Manal et al., 2015). We therefore elected to quantify medial knee joint loading using a model that considers the KAM and the KFM as well as the timing of muscle activity within the gait 97 cycle that contributes to these moments (Schipplein \& Andriacchi, 1991).

98

\section{MATERIALS \& METHODS}

100

101

102

103

104 105 106 108

\section{Subjects}

The study design was cross-sectional, with a "limb loss" group and a "control" group.

The limb loss group consisted of 10 service members with unilateral limb loss. Five subjects had transtibial amputations and five had transfemoral amputations. The descriptive statistics of the limb loss group (mean \pm SD) were: age $27 \pm 5$ years, height $1.77 \pm 0.05 \mathrm{~m}$, mass $81.1 \pm 18.4 \mathrm{~kg}$, and 170(36) days from their most recent amputation-related surgery. All subjects were male and had been walking independently without assistive devices other than their prosthesis for an average of two months at the time of data collection. Additional inclusion criteria were no previous diagnosis of OA, no pain during activities of daily living greater than 4 on a 10-point scale, no limb loss elsewhere on the body, no history of traumatic injury to the intact limb, and no history of traumatic brain injury or other medical issues known to affect gait.

11 The control group consisted of 10 male service members with no limb loss and similar descriptive statistics to the limb loss group (age $30 \pm 4$ years, height $1.79 \pm 0.07 \mathrm{~m}$, mass $83.8 \pm 14.3$ 
$113 \mathrm{~kg}$ ), who also met all the inclusion criteria. Walter Reed National Military Medical Center

114 granted ethical approval to carry out the study within its facilities (IRB reference number

115 350985). All protocols were approved by the ethics committee. All subjects were briefed on the

116 study protocols and gave informed written consent prior to participating.

118 Experimental Setup

119 An instrumented gait analysis was performed while subjects walked across a level 15-m

120 walkway. Subjects wore shorts and their own athletic footwear. The limb loss subjects used

121 their own clinically prescribed passive prosthesis. Positions of 23 retroreflective markers on the

122 pelvis and lower limbs were sampled at $120 \mathrm{~Hz}$ using 23 optical motion capture cameras (Vicon,

123 Oxford, UK). Ground reaction forces (GRF) were sampled synchronously at $1200 \mathrm{~Hz}$ using six

124 force platforms (AMTI, Watertown, MA, USA) embedded in the walkway. Individual markers

125 were attached by double-sided tape on the anterior- and posterior-superior iliac spines, iliac

126 crests, greater trochanters, medial and lateral femoral epicondyles, medial and lateral malleoli,

127 heads of the 2nd and 5th metatarsals, and heel of the shoe. Lightweight shells with clusters of

128 four markers were attached to the thigh and shank using elastic wraps. The medial markers were

129 removed after a standing calibration trial and were reconstructed as virtual markers during the

130 walking trials.

131

132 Protocol

133 Subjects walked along the walkway at self-selected speed and cadence. Instructions were

134 to walk in a "normal and comfortable" fashion. Each subject walked back and forth along the

135 walkway until five acceptable trials were collected, with "acceptable" defined as each foot 
136 contained entirely within the bounds of a single force platform and both feet never

137 simultaneously contacting the same platform.

138

139 Data Processing

140 Marker positions and GRF from each trial were exported to Visual3D (C-Motion,

141 Germantown, MD, USA) for further analysis. Marker positions and GRF were smoothed using a 142 4th-order dual-pass Butterworth filter with cutoff frequencies of $6 \mathrm{~Hz}$ and $50 \mathrm{~Hz}$, respectively. A

143 linked-segment model of each subject's pelvis and intact limb was defined using marker

144 positions from a standing calibration trial. The hip joint center was estimated from the positions

145 of the pelvis markers (Bell et al., 1989). The knee center was estimated as the midpoint of the

146 femoral condyle markers, and the ankle joint center was estimated as the midpoint of the malleoli

147 markers. The long axes of the thigh and shank were defined between the proximal and distal

148 joint centers. The frontal plane axis for both segments was defined from the cross-product of the

149 long axis and the vector between the femoral condyles. The sagittal plane axis was the cross-

150 product of the frontal plane and long axes. Segment tracking during gait trials was calculated

151 from the positions of marker clusters on rigid shells.

152 Joint angles during gait were calculated using 6DOF pose estimation, with a Cardan $X y z$

153 rotation sequence (Wu \& Cavanagh, 1995). Resultant joint forces and moments were calculated

154 by iterative Newton-Euler inverse dynamics beginning at the foot (Selbie et al., 2014). The

155 resultant knee forces and moments were expressed in the shank reference frame.

156

157 Joint Contact Force Modeling 
Andriacchi (1991). Muscle moment arms and orientations were defined as quadratic functions of

160 the knee flexion angle using average values for men from Wretenberg et al. (1996). The KFM

161 was assumed to be produced by the quadriceps if the moment was extensor, by the hamstrings if

162 the moment was flexor in swing or during early stance, and by the gastrocnemius if the moment

163 was flexor in late stance. Forces in the individual hamstrings muscles (biceps femoris,

164 semimembranosus, semitendinosus) and the two gastrocnemius heads were distributed by the

165 ratios of their physiological cross-sectional areas from Arnold et al. (2010). The medial contact

166 force was then calculated by balancing the frontal plane moments about the lateral contact point

167 (Fig. 1). Cruciate and collateral ligament forces were included in the contact force calculation

168 using the method described by Morrison (1968). The distance between the medial and lateral

169 tibiofemoral contact points in the frontal plane was assumed to be $5.0 \mathrm{~cm}$ on average and was

170 scaled linearly for each subject by the distance between the medial and lateral femoral condyle

171 markers during the standing calibration trial. Baseline model parameters are summarized in

172 Table 1.

173 Note that this model assumes zero antagonistic co-contraction. This assumption could

174 potentially underestimate contact forces around heel-strike, when the quadriceps and hamstrings

175 are both active (Sutherland, 2001). However, since knee muscle co-contraction in early stance is

176 similar between limb loss subjects and controls (Seyedali et al., 2012), this assumption does not

177 bias the results in favor of the hypothesis.

178

179 Statistical Analysis 
181 between groups. These outcome variables were scaled by bodyweight (BW), with the mass of

182 the prosthesis included in this calculation for the limb loss subjects. Loading rate was defined as

183 the maximum loading rate during $10-90 \%$ of the time from heel-strike until the first peak.

184

Results will be presented for the transtibial and transfemoral subjects separately.

185 However, due to the small sample sizes, these subjects were combined into a single limb loss

186 group for statistical comparison with the control group. It will be seen that the differences in

187 contact forces between the limb loss and control groups were not driven by the transtibial or

188 transfemoral subjects specifically (i.e. contact forces were similar on average for transtibial and

189 transfemoral subjects).

190 Normality of the outcome variables was assessed using Kolmogorov-Smirnov tests. All

191 tests passed at the $\alpha=0.05$ level. Subsequently, comparisons between groups were made using

192 independent Student's $t$-tests $(\alpha=0.05, \beta=0.20)$ with a False Discover Rate adjustment for the

193 multiple outcome variables. The tests were one-tailed due to the directional nature of the

194 hypothesis. 95\% confidence intervals (CI) were also calculated. As an additional conservative

195 check due to the small sample sizes, differences were reported only if the effect size was large

196 (Cohen's $d>0.80$ ). Effect sizes for between-subjects differences in external knee adduction

197 moment (a common surrogate for medial joint loading) and knee OA initiation and progression

198 are typically much smaller than 0.8 (e.g. Amin et al., 2004; Miyazaki et al., 2002), so the

199 requirement of a large effect size is likely a fairly conservative check.

200

201 Sensitivity Analysis 
203 and physiological cross-sectional areas, and the distance between the tibiofemoral contact points.

204 The necessary imaging data to define these parameters on a subject-specific basis were not

205 available, and the same generic parameter values were used for all subjects except for the contact

206 point distance. In such situations, probabilistic approaches are useful for assessing the sensitivity

207 of model output to parameter value uncertainty (Valero-Cuevas et al., 2009). To assess the

208 sensitivity of the contact force results (and the conclusions drawn from them) to these parameter

209 values, standard normal distributions were formed for each parameter with the nominal value as

210 the mean and a coefficient of variation of $10 \%$, which is a reasonable estimate of the typical

211 variation in these parameters in a homogenous adult male population (Hasson \& Caldwell,

212 2012). The contact force variables were then re-calculated for each subject using parameters

213 randomly drawn from these distributions, and the statistical analysis was performed again. This

214 process was repeated iteratively until the fraction of iterations with significantly greater outcome

215 variables in the limb loss group changed by under $1 \%$ over 100 further iterations. The output of

216 this analysis was the fraction of perturbed parameter sets for which the outcome variable in

217 question (peak, loading rate, or impulse) was greater in the limb loss group, from which the

218 sensitivity of the outcome variables to the assumed model parameters could be judged.

\section{RESULTS}

Subject-specific data including descriptors, outcome variables, and waveforms of knee

222 joint kinetics, kinematics, and medial contact forces, are included in the supplementary material.

223 The self-selected walking speeds were similar between groups $(1.25 \pm 0.19 \mathrm{~m} / \mathrm{s}$ for limb loss,

$2241.31 \pm 0.10 \mathrm{~m} / \mathrm{s}$ for controls, $p=0.40, d=0.39,95 \% \mathrm{CI}=(-0.19,0.07) \mathrm{m} / \mathrm{s})$. Stride durations were 
225 also similar between groups $(1.16 \pm 0.07 \mathrm{~s}$ for limb loss, $1.12 \pm 0.07 \mathrm{~s}$ for controls, $p=0.24, d=$

$2260.55,95 \% \mathrm{CI}=(-0.02,0.10) \mathrm{s})$. The average medial knee joint contact force waveforms are

227 shown for the transtibial, transfemoral, and control subjects in Fig. 2. The contact forces showed

228 the typical two-peaked pattern seen in instrumented knee replacement studies of older adults

229 without limb loss (Walter et al., 2010; Kutzner et al., 2013; Meyer et al., 2013). For the control

230 subjects, the peak force occurred in early stance and averaged $1.57 \pm 0.26 \mathrm{BW}$, which is within

231 the range of values reported in these studies (1.25-2.20 BW). With the exception of a lack of

232 quadriceps activity in late swing, which did not affect the contact force outcome variables, the

233 muscle forces predicted by the model for the quadriceps, hamstrings, and gastrocnemius (Fig. 3)

234 were consistent with normative electromyogram timing for these muscles (Sutherland, 2001;

235 Seyedali et al., 2012).

236 The peak contact force was greater in the limb loss group than in the control group

$237(1.84 \pm 0.37$ vs. $1.57 \pm 0.26 \mathrm{BW}, p=0.037, d=0.85,95 \% \mathrm{CI}=(-0.01,0.55) \mathrm{BW})$. Maximum

238 loading rate was also greater in the limb loss group $(24.7 \pm 5.4 \mathrm{vs} .19 .9 \pm 3.2 \mathrm{BW} / \mathrm{s}, p=0.012, d=$

$2391.10,95 \% \mathrm{CI}=(1.0,8.7) \mathrm{BW} / \mathrm{s})$. Impulse had a moderate effect size between groups, but were

240 not significantly greater in the limb loss group $(0.72 \pm 0.12 \mathrm{BW} \cdot \mathrm{s}$ for limb loss, $0.64 \pm 0.13 \mathrm{BW} \cdot \mathrm{s}$

241 for controls, $p=0.084, d=0.64,95 \% \mathrm{CI}=(-0.03,0.19) \mathrm{BW} \cdot \mathrm{s})$. Outcome variables are

242 summarized in Fig. 4. The sensitivity analysis converged after about 3,000 iterations (Fig. 5).

243 The loading rate, peak, and impulse were greater in the limb loss group than in the control group $244(p<0.05, d>0.80)$ for $98 \%, 73 \%$, and $25 \%$ of these iterations, respectively.

245 The KAM and KFM were not analyzed statistically due to concerns over multiple 246 comparisons with small sample sizes, and the fact that both variables were considered in the 247 calculation of contact forces, but their mean profiles are presented for completeness in Fig. 6. 
248 Limb loss subjects tended to have greater peak KFM and KAM than the control subjects, and the

249 transtibial subjects tended to have greater peak KFM than the transfemoral subjects. The

250 primary mechanism by which the transtibial and transfemoral subjects had similar peak contact

251 forces (Fig. 2) despite greater KFM in the transtibial subjects was greater axial resultant joint

252 force in the transfemoral subjects during early stance (Fig. 6).

253

254 DISCUSSION

255 In this study we tested the hypothesis that knee joint loading during walking is greater in

256 the intact limb of US Military service members with unilateral limb loss who are relatively

257 young, recently ambulatory, and otherwise healthy, compared to the limbs of service members

258 with similar demographics and no limb loss. Based on the nominal contact force results (Fig. 4)

259 and the probabilistic analysis of model parameters (Fig. 5), we accept this hypothesis with a high

260 degree of confidence based on the loading rate of the medial joint contact force, and with a

261 moderate degree of confidence based on the peak of the medial joint contact force. Impulse of

262 the contact force did not appear to be greater in the limb loss group.

263 Before discussing the implications of these results, we first comment on some limitations.

264 The study included small sample sizes, with a mix of transtibial and transfemoral subjects (five

265 each) in the limb loss group. However, the transtibial and transfemoral subjects had similar

266 average height, mass, and self-selected walking speeds (differences of $0.4 \mathrm{~cm}, 2.8 \mathrm{~kg}$, and 0.02

$267 \mathrm{~m} / \mathrm{s}$, respectively), and it can be seen from Fig. 4 that they also had similar contact force results,

268 suggesting that combining these sub-groups into one group was reasonable for the purposes of

269 this study. The limb loss population is difficult to study in large numbers, and we were working

270 with a particular subset of this population (young service members and some fairly restrictive 
271 additional inclusion criteria). Due to the small sample size, we took a conservative approach to

272 reporting differences between groups even after adjusting for multiple comparisons, with the

273 requirement of a large effect size. The issues of the knee model parameters and antagonistic co-

274 contraction have already been addressed: these modeling issues may affect the numerical values

275 of the results, but would be unlikely to change the conclusions (Fig. 5). The knee contact model

276 itself (Fig. 1) is a Morrison (1968)-type reduction approach. These models are on the lower end

277 of complexity among the range of musculoskeletal models used for this purpose, but have a long

278 history in biomechanics (Morrison, 1968; Schipplein \& Andriacchi, 1991; DeVita \& Hortobágyi,

279 2001; Messier et al., 2011; Willy et al., 2016). History/popularity alone do not validate the

280 approach, but this approach produces similar muscle forces to more mathematically intensive

281 static optimization methods (Kernozek et al., 2016) and knee contact forces in good agreement

282 with instrumented knee replacement measurements (Willy et al., 2016).

283 The time point at which the gait data were obtained from the limb loss group (shortly

284 after they became independently mobile) could be viewed as a limitation since the gait

285 mechanics of these individuals may change in the future. Although the knee kinetics in the

286 present limb loss subjects (Fig. 6) are similar to those in studies on more experienced prosthesis

287 users (Royer \& Koenig, 2005; Russell Esposito \& Wilken, 2014), the data here may not

288 represent the "typical" or "average" loads these subjects will experience later in life, due for

289 example to motor learning, experience, changes in fitness, or use of different prostheses.

290 However, the focus on joint loading early on in the rehabilitation process can also be viewed as a

291 strength of the present study. Human articular cartilage appears to undergo at least some degree

292 of structural and functional atrophy in the absence of mechanical loading, and it is unclear if

293 these changes are fully reversible (Vanwanseele et al., 2002; Hudelmaeir et al., 2006; Souza et 
294 al., 2012; Owman et al., 2014). When new prosthesis users first begin walking independently, 295 their joints have likely undergone a period of at least several weeks with no or minimal 296 mechanical loading following injury, surgery, and recovery. At this early time, we speculate that 297 placing abnormal loads on the intact limb may be particularly dangerous for the future health of 298 the knee. To minimize this risk, we suggest that long periods of unloading should be avoided to 299 the extent that doing so is safe and feasible for the patient. To the knowledge of the authors, the present study is the first to show that medial knee joint contact forces were greater in the intact limb than in controls. A recent forward dynamics simulation study showed a similar result for the total joint contact force during walking in individuals with unilateral transtibial limb loss (Silverman \& Neptune, 2014). Knee OA has a rising incidence among young United States military service members over the past 10 years, 305 and there is a need to develop more effective preventive strategies in at-risk sub-groups of this population (Showery et al., 2016). There are presently no longitudinal studies on baseline joint loading and the initiation of knee OA in the limb loss population. However, Morgenroth et al. (2014) found that the KAM peak, impulse, and loading rate were all significantly correlated with the degree of knee structural abnormality present in the intact limb of middle-aged adults (mean age 56 years) with unilateral transfemoral amputations. The present results suggest that relatively high loads are present on the medial knee of the intact limb when young service members with limb loss begin to walk independently, and when interpreted in light of

313 Morgenroth et al. (2014), that the long-term consequence of these loads may be structural 314 degeneration of the knee. These suggestions are in need of verification in longitudinal studies. Relatedly, while the present results suggest medial knee joint loading was greater in the 316 limb loss group, the size of the "minimum meaningful difference" that actually affects the risk 
317 for knee OA is unknown. Studies using the external knee adduction moment suggest that effect

318 sizes for differences in medial joint loading during walking and the initiation and progression

319 knee OA in older adults may be small (Amin et al., 2004; Miyazaki et al., 2002), but it is

320 unknown if this suggestion generalized to actual medial joint contact forces or to a younger

321 military limb loss population. Two recent studies suggest that the "minimum detectable change"

322 in medial knee joint loading from gait modification is about $0.25-0.30 \mathrm{BW}$ for peak and about

$3230.04 \mathrm{BW} \bullet$ s for impulse (Gardinier et al., 2013; Barrios \& Willson, 2016). Those data were from

324 within-subject designs, where the present data are between-subjects, but they suggest that

325 differences smaller than these values may be difficult to reliably detect in gait analysis, even if

326 they are biologically meaningful. For reference, the average differences between the limb loss

327 and control results in the present study were $0.27 \mathrm{BW}$ for peak and $0.08 \mathrm{BW} \bullet \mathrm{s}$ for impulse.

328 Additional knowledge from longitudinal studies is needed to understand which features of joint

329 loading and cartilage mechanics are most important for predicting future structural degeneration, 330 and if critical thresholds for those variables exist.

As noted earlier, the KAM is presently the most popular variable for assessing medial

332 knee joint loading in human gait. While we did not analyze the KAM statistically due to

333 concerns over the small sample sizes and multiple comparisons, visual inspection of the KAM

334 (Fig. 6) suggests that similar conclusions would have been reached had we used the KAM rather 335 than the medial joint contact force as the primary outcome variable: greater peak and greater 336 loading rate in the limb loss group. However, we caution that this result was likely coincidental 337 and is not a mechanical requirement. The KAM alone does not dictate the loading of the medial 338 knee, as recent instrumented knee implant studies have shown (Walter et al., 2010; Kutzner et 339 al., 2013; Meyer et al., 2013). Relatedly, the KFM has a major influence on the shape, 
340 magnitude, and medial/lateral ratio of joint contact forces, and should be considered when

341 assessing joint loading in gait (Manal et al., 2015).

342

\section{CONCLUSIONS}

344 In summary, the present results suggest that young, recently ambulatory service members

345 with unilateral limb loss place relatively high loads on their medial knee when walking compared

346 to controls without limb loss. We suggest these loads may be a risk factor for future

347 development of knee OA, a common secondary condition in this population. Further

348 longitudinal study and development of preventive interventions (e.g. physical activity guidelines,

349 prosthesis designs) is warranted. The results also indicate that knee contact model parameter

350 values can be an important consideration in cross-sectional studies. Here we investigated the

351 overall sensitivity (perturbing all contact model parameters simultaneously), but sensitivity to

352 particular parameters of interest may be a relevant topic for future work or specific applications.

353

354 ACKNOWLEDGEMENTS

355 The authors would like to thank Dr. Kurt Manal for helpful suggestions on calculating the

356 knee joint contact forces and Mrs. Jenna Trout for her assistance with data collection and

357 processing.

358

359

360

361

362

363

364

365

366

\section{REFERENCES}

Amin S, Lueopongsak N, McGibbon CA, LaValley MP, Krebs DE, and Felson DT (2004).

Knee adduction moment and development of chronic knee pain in elders. Arthritis \& Rheumatism 51, 371-376.

Andriacchi TP and Mündermann A (2006). The role of ambulatory mechanics in the initiation and progression of knee osteoarthritis. Current Opinion in Rheumatology 18, 514-518. 
367 Arnold EM, Ward SR, Lieber RL, and Delp SL (2010). A model of the lower limb for analysis 368 of human movement. Annals of Biomedical Engineering 38, 269-279.

369

370

371

372

373

374

375

376

377

378

379

380

381

382

383

384

385

386

387

388

389

390

391

392

393

394

395

396

397

398

399

400

401

402

403

404

405

406

407

408

409

410

411

Arokoski JPA, Jurvelin JS, Väätäinen U, and Helminen HJ (2000). Normal and pathological adaptations of articular cartilage to joint loading. Scandinavian Journal of Medicine \& Science in Sports 10, 186-198.

Barrios J and Willson J (2016). Minimum detectable change in medial tibiofemoral contact force parameters: derivation and application to a load-altering intervention. Journal of Applied Biomechanics, doi: 10.1123/jab.2016-0163.

Bell AL, Brand RA, and Peterson DR (1989). Prediction of hip joint centre location from external landmarks. Human Movement Science 8, 3-16.

Bellucci G and Seedhom BB (2001). Mechanical behaviour of articular cartilage under tensile cyclic load. Rheumatology 40, 1337-1345.

Borgmann F (1960). Zur gutachtlichen beurteilung von ruckenbeschwerden und befunden bei oberschelamputation. Zeitschrift für Orthopädie und ihre Grenzgebiete 93, 351-64.

Centers for Disease Control and Prevention (2015). Osteoarthritis. Retrieved from: http://www.cdc.gov/arthritis/basics/osteoarthritis.htm, June 6, 2016.

DeVita P and Hortobágyi T (2001). Functional knee brace alters predicted knee muscle and joint forces in people with ACL reconstruction during walking. Journal of Applied Biomechanics 17, 297-311.

Esposito ER and Wilken JM (2014). Biomechanical risk factors for knee osteoarthritis when using passive and powered ankle-foot prostheses. Clinical Biomechanics 29, 1186-1192.

Farrokhi S, Mazzone B, Yoder A, Grant K, and Wyatt M (2016). A narrative review of the prevalence and risk factors associated with development of knee osteoarthritis after traumatic unilateral lower limb amputation. Military Medicine 181, 38-44.

Felson DT (2013). Osteoarthritis as a disease of mechanics. Osteoarthritis \& Cartilage 21, 1015.

Fischer H (2015). A Guide to US Military Casualty Statistics: Operation Freedom's Sentinel, Operation Inherent Resolve, Operation New Dawn, Operation Iraqi Freedom, and Operation Enduring Freedom. Washington, DC: Congressional Research Service 7-5700.

Foroughi N, Smith R, and Vanwanseele B (2009). The association of external knee adduction moment with biomechanical variables in osteoarthritis: a systematic review. The Knee 16, 303309. 
412 Gailey R, Allen K, Castles J, Kucharik J, and Roeder M (2008). Review of secondary physical 413 conditions associated with lower-limb amputation and long-term prosthesis use. Journal of 414 Rehabilitation Research \& Development 45, 15-30.

415

Gardinier ES, Manal K, Buchanan TS, and Snyder-Mackler L (2013). Minimum detectable change for knee joint contact force estimates using an EMG-driven model. Gait \& Posture 38, 1051-1053.

Hasson CJ and Caldwell GE (2012). Effects of age on mechanical properties of dorsiflexor and plantarflexor muscles. Annals of Biomedical Engineering 40, 1088-1101.

Hudelmaier M, Glaser C, Hausschild A, Burgkart R, and Eckstein F (2006). Effects of joint loading and reloading on human cartilage morphology and function, muscle cross-sectional areas, and bone density - a quantitative case report. Journal of Musculoskeletal \& Neuronal Interactions 6, 284-290.

Hungerford D and Cockin J (1975). Fate of the retained lower limb joints in second World War amputees. Journal of Bone \& Joint Surgery 57, 111.

Jurvelin J, Kiviranta I, Tammi M, and Helminen HJ (1986). Softening of canine articular cartilage after immobilization of the knee joint. Clinical Orthopaedics 201, 246-252.

Kernozek T, Gheidi N, and Ragan R (2016). Comparison of estimates of Achilles tendon loading from inverse dynamics and inverse dynamics-based static optimisation during running. Journal of Sports Sciences, doi: 10.1080/02640414.2016.1255769.

Kutzner I, Trepczynski A, Heller MO, and Bergmann G (2013). Knee adduction moment and medial contact force: facts about their correlation during gait. PLOS ONE 8, e81036.

Lemaire ED and Fisher FR (1994). Osteoarthritis and elderly amputee gait. Archives of Physical Medicine \& Rehabilitation 75, 1094-1099.

Maly MR (2008). Abnormal and cumulative loading in knee osteoarthritis. Current Opinion in Rheumatology 20, 547-552.

Manal K, Gardinier E, Buchanan TS, and Snyder-Mackler L (2015). A more informed evaluation of medial compartment loading: the combined use of the knee adduction and flexor moments. Osteoarthritis \& Cartilage 23, 1107-1111.

Messier SP, Legault C, Loeser RF, Van Arsdale SG, Davis C, Ettinger WH, and DeVita P (2011). Does high weight loss in older adults with knee osteoarthritis affect bone-on-bone joint loads and muscle forces during walking? Osteoarthritis \& Cartilage 19, 272-280.

Meyer AJ, D'Lima DD, Besier TF, Lloyd DG, Colwell CW, and Fregly BJ (2013). Are external knee load and EMG measures accurate indicators of internal knee contact forces during gait? Journal of Orthopaedic Research 31, 921-929. 
458

459

460

461

462

463

464

465

466

467

468

469

470

471

472

473

474

475

476

477

478

479

480

481

482

483

484

485

486

487

488

489

490

491

492

493

494

495

496

497

498

499

500

501

Miller RH, Edwards WB, Brandon SCE, Morton AM, and Deluzio KJ (2014). Why don't most runners get knee osteoarthritis? A case for per-unit-distance loads. Medicine \& Science in Sports \& Exercise 46, 572-579.

Miller RH, Esterson AY, and Shim JK (2015). Joint contact forces when minimizing the external knee adduction moment by gait modification: a computer simulation study. The Knee 22, 481-489.

Miyazaki T, Wada M, Kawahara H, Sato M, Baba H, and Shimada S (2002). Dynamic load at baseline can predict radiographic disease progression in medial compartment knee osteoarthritis. Annals of the Rheumatic Diseases 61, 617-622.

Morgenroth DC, Gellhorn AC, and Suri P (2012). Osteoarthritis in the disabled population: a mechanical perspective. Physical Medicine \& Rehabilitation 4, S20-S27.

Morgenroth DC, Medverd JR, Seyedali M, and Czerniecki JM (2014). Relationship between knee joint loading rate during walking and degenerative changes on magnetic resonance imaging. Clinical Biomechanics 29, 664-670.

Morrison JB (1968). Bioengineering analysis of force actions transmitted by the knee joint. Biomedical Engineering 3, 164-170.

Norvell DC, Czerniecki JM, Reiber GE, Maynard C, Pecoraro JA, and Weiss NS (2005). The prevalence of knee pain and symptomatic knee osteoarthritis among veteran traumatic amputees and nonamputees. Archives of Physical Medicine \& Rehabilitation 86, 487-493.

Owman H, Tiderius CJ, Ericsson YB, and Dahlberg LE (2014). Long-term effect of removal of knee joint loading on cartilage quality evaluated by delayed gadolinium-enhanced magnetic resonance imaging of cartilage. Osteoarthritis \& Cartilage 22, 928-932.

Pruziner AL, Werner KM, Copple TJ, Hendershot BD, and Wolf EJ (2014). Does intact limb loading differ in Servicemembers with traumatic lower limb loss? Clinical Orthopaedics \& Related Research 472, 3068-3075.

Royer T and Koenig M (2005). Joint loading and bone mineral density in persons with unilateral, trans-tibial amputation. Clinical Biomechanics 20, 1119-1125.

Schipplein OD and Andriacchi TP (1991). Interaction between active and passive knee stabilizers during level walking. Journal of Orthopaedic Research 9, 113-119.

Seedhom BB (2006). Conditioning of cartilage during normal activities is an important factor in the development of osteoarthritis. Rheumatology 45, 146-149. 
502 Selbie WS, Hamill J, and Kepple T (2014). Three-dimensional kinetics. In: Robertson DGE, 503 Caldwell GE, Hamill J, Kamen G, and Whittlesey SN (eds.), Research Methods in Biomechanics $5042^{\text {nd }}$ Edition (pp. 151-176). Champaign: Human Kinetics.

505

506

507

508

509

510

511

512

513

514

515

Seyedali M, Czerniecki JM, Morgenroth DC, and Hahn ME (2012). Co-contraction patterns of trans-tibial ankle and knee musculature during gait. Journal of NeuroEngineering \& Rehabilitation 9, 29.

Showery JE, Kusnezov NA, Dunn JC, Bader JO, Belmont PJ, and Waterman BR (2016). The rising incidence of degenerative and posttraumatic osteoarthritis of the knee in the United States military. Journal of Arthroplasty, doi: 10.1016/j.arth.2016.03.026.

Silverman AK and Neptune RR (2014). Three-dimensional knee joint contact forces during walking in unilateral transtibial amputees. Journal of Biomechanics 47, 2556-2562.

Simic M Hinman RS, Wrigley TV, Bennell KL, and Hunt MA (2011). Gait modification strategies for altering medial knee joint load: a systematic review. Arthritis Care \& Research 63, 405-426.

Souza RB, Baum T, Wu S, Feeley BT, Kadel N, Li X, Link TM, and Majumdar S (2012). Effects of unloading on knee articular cartilage T1rho and T2 magnetic resonance imaging relaxation times: a case series. Journal of Orthopaedic \& Sports Physical Therapy 42, 511-520.

Sutherland DH (2001). The evolution of clinical gait analysis part 1: kinesiological EMG. Gait \& Posture 14, 61-70.

Terzidis I, Totlis T, Papathanasiou E, Sideridis A, Vlasis K, and Natsis K (2012). Gender and side-to-size differences of femoral condyles morphology: osteometric data from 360 Caucasian dried femori. Anatomy Research International 2012, 679658.

Valero-Cuevas FJ, Hoffmann H, Kurse MU, Kutch JJ, and Theodorou EA (2009). Computational models for neuromuscular function. IEEE Reviews in Biomedical Engineering 2, $110-135$.

Vanwanseele B, Eckstein F, Knecht H, Stüssi E, and Spaepen A (2002). Knee cartilage of spinal cord-injured patients displays progressive thinning in the absence of normal joint loading and movement. Arthritis \& Rheumatism 46, 2073-2078.

Walter JP, D'Lima DD, Colwell CW, and Fregly BJ (2010). Decreased knee adduction moment does not guarantee decreased medial contact force during gait. Journal of Orthopaedic Research 28, 1348-1354.

Weightman B, Chappell DJ, and Jenkins EA (1978). A second study on the tensile fatigue properties of human articular cartilage. Annals of the Rheumatic Diseases 37, 58-63. 
547 Willy RW, Meardon SA, Schmidt A, Blaylock NR, Hadding SA, and Willson JD (2016).

548 Changes in tibiofemoral contact forces during running in response to in-field gait retraining.

549 Journal of Sports Sciences 34, 1602-1611.

550

551 Wise BL, Niu J, Yang M, Lane NE, Harvey W, Felson DT, Hietpas J, Nevitt M, Sharma L, 552 Torner J, Lewis CE, and Zhang Y (2012). Patterns of compartment involvement in tibiofemoral 553 osteoarthritis in men and women and in Caucasians and African Americans. Arthritis Care \& 554 Research 64, 847-852.

555

556 Wretenberg P, Németh G, Lamontagne M, and Lundin B (1996). Passive knee muscle moment 557 arms measured in vivo with MRI. Clinical Biomechanics 11, 439-446.

558

$559 \mathrm{Wu} \mathrm{G}$ and Cavanagh PR (1995). ISB recommendations for standardization in the reporting of 560 kinematic data. Journal of Biomechanics 28, 1257-1261. 


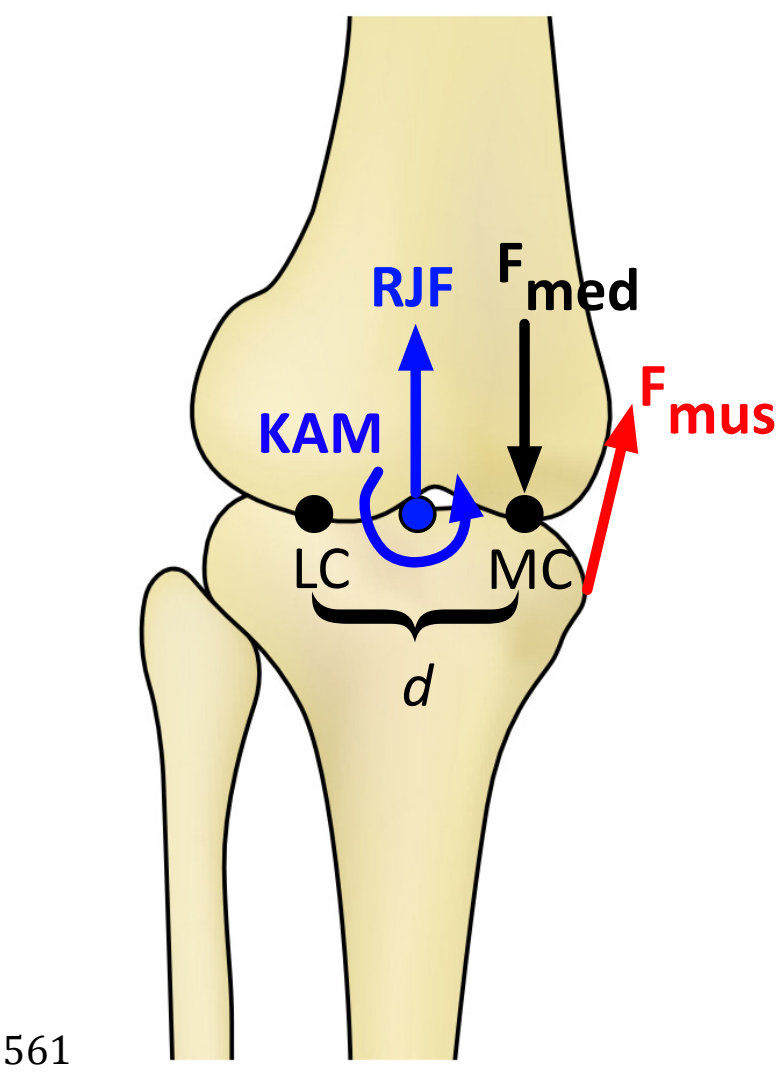

562 Figure 1. Schematic of the knee model in the frontal plane for calculating the medial knee joint 563 contact force $\left(\mathrm{F}_{\mathrm{med}}\right)$. KAM $=$ knee adduction moment; $\mathrm{RJF}=$ resultant axial joint force; $\mathrm{F}_{\mathrm{mus}}=$ muscle force, determined by the knee flexion moment; $\mathrm{LC}$ and $\mathrm{MC}=$ medial and lateral contact points, separated by distance $d$. $\mathrm{F}_{\text {med }}$ is calculated by balancing the moments produced about the 566 point LC (Schipplein \& Andriacchi, 1991). 


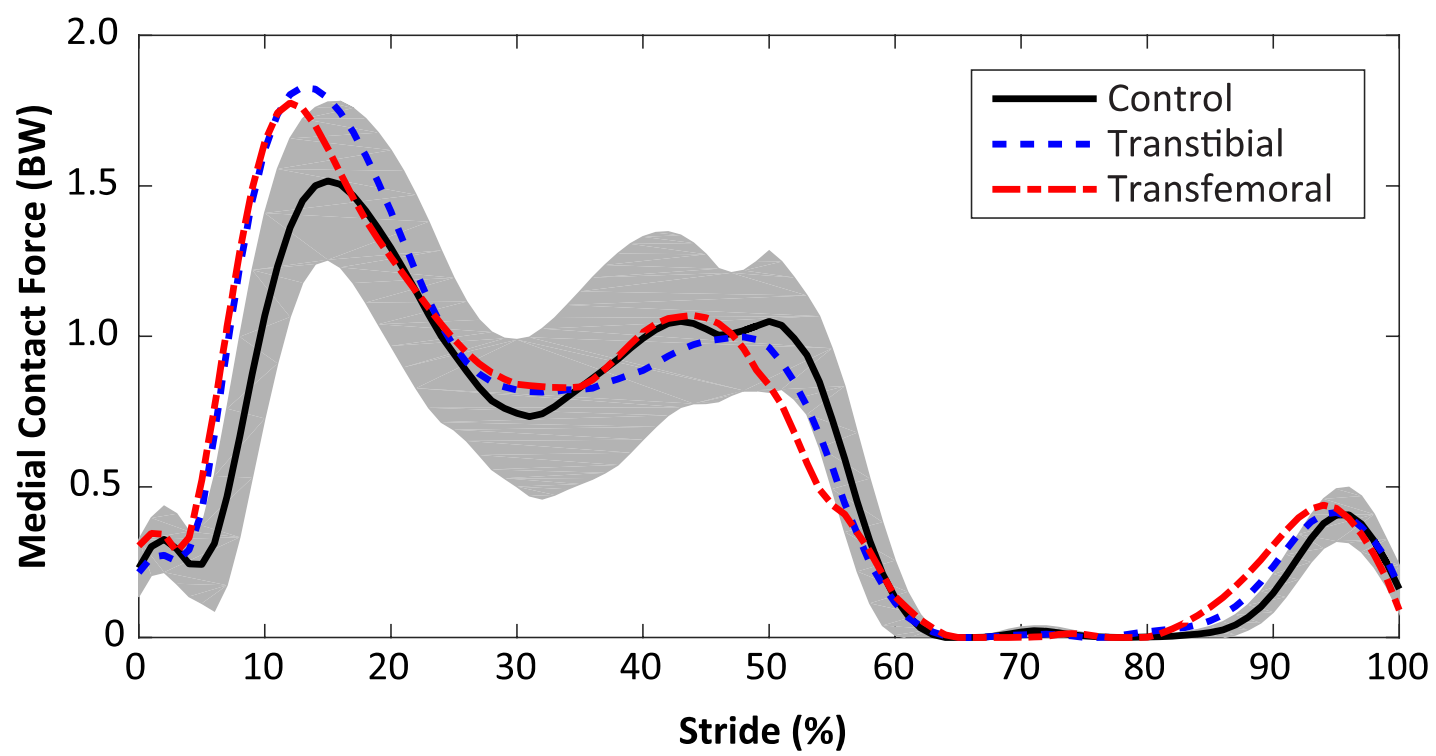

Figure 2. Medial knee joint contact forces in percent bodyweight (BW) during the stride,

569 beginning at heel-strike. Solid, dashed, and dash-dotted lines are means for control, transtibial, 570 and transfemoral subjects. The shaded areas are \pm one between-subjects standard deviation for 571 the control subjects. 

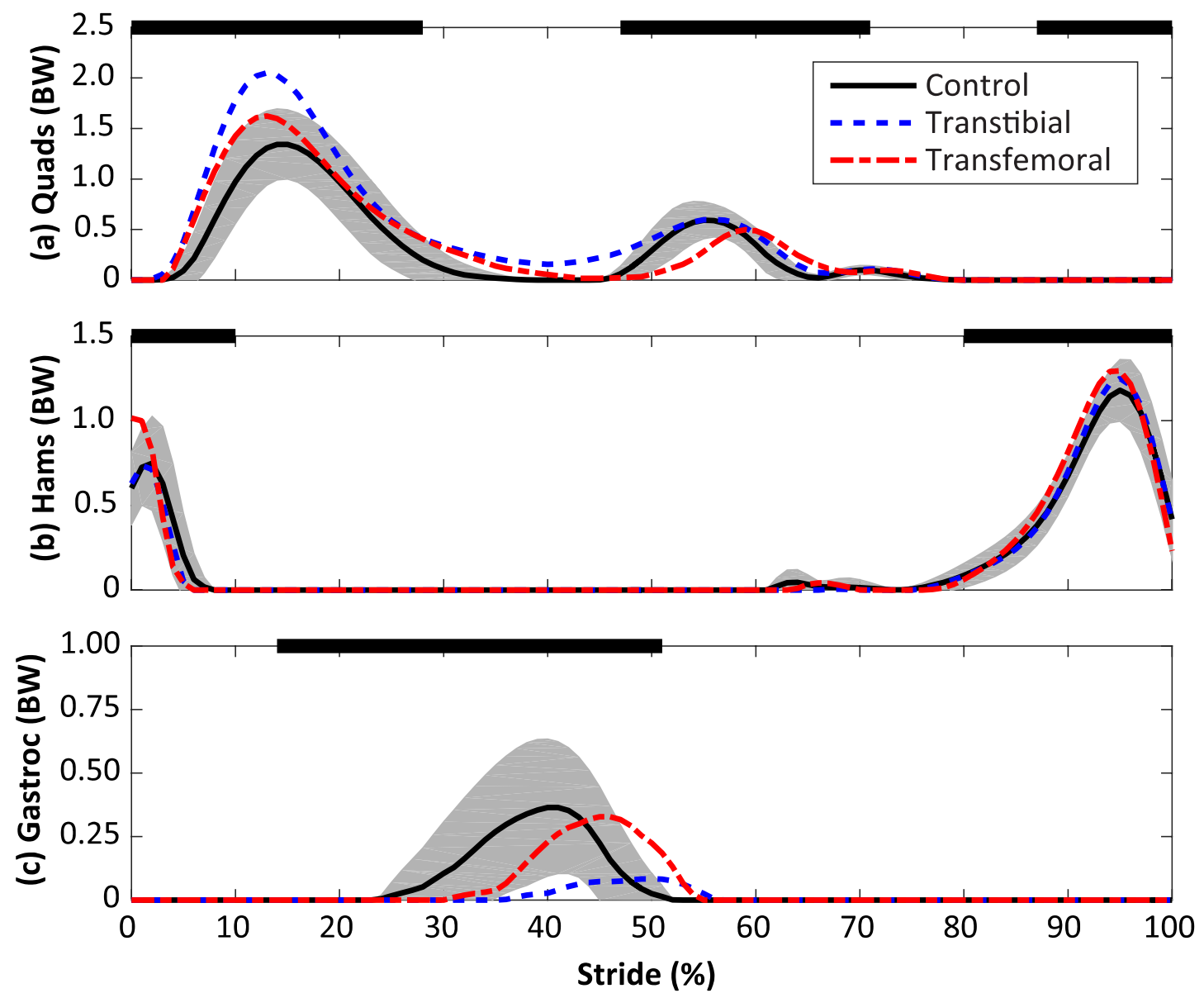

Figure 3. Calculated muscle forces for the quadriceps (Quads, a), hamstrings (Hams, b), and gastrocnemius (Gastroc, c) muscles during the stride, beginning at heel-strike. Solid, dashed, and dash-dotted lines are means for control, transtibial, and transfemoral subjects. The shaded areas are \pm one between-subjects standard deviation for the control subjects. Scaling factors were bodyweight (BW). The black bars along the top of each panel denote the fraction(s) of the gait cycle when this muscle group is "on" according to normative electromyograms (Sutherland, 2001), which are similar for the intact limb in limb loss subjects (Seyedali et al., 2012). 

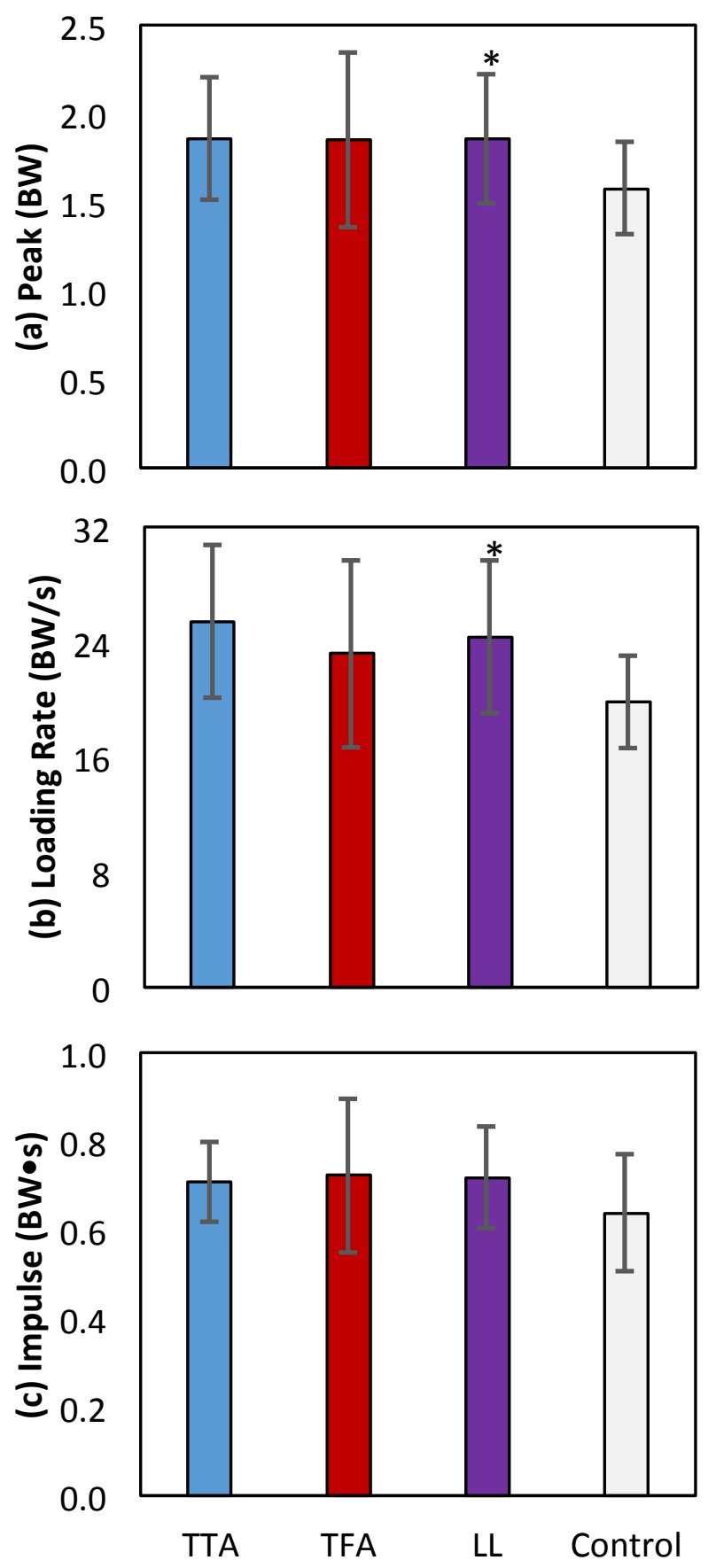

Figure 4. Means \pm one between-subjects standard deviation for peak (a), loading rate (b), and impulse (c) of the medial knee joint contact force for the transtibial subjects (TTA), the

583 transfemoral subjects (TFA), and the control subjects. The limb loss bars (LL) are data for the 584 TTA and TFA subjects combined. * = greater than control, with a large effect size $(d>0.80)$. 


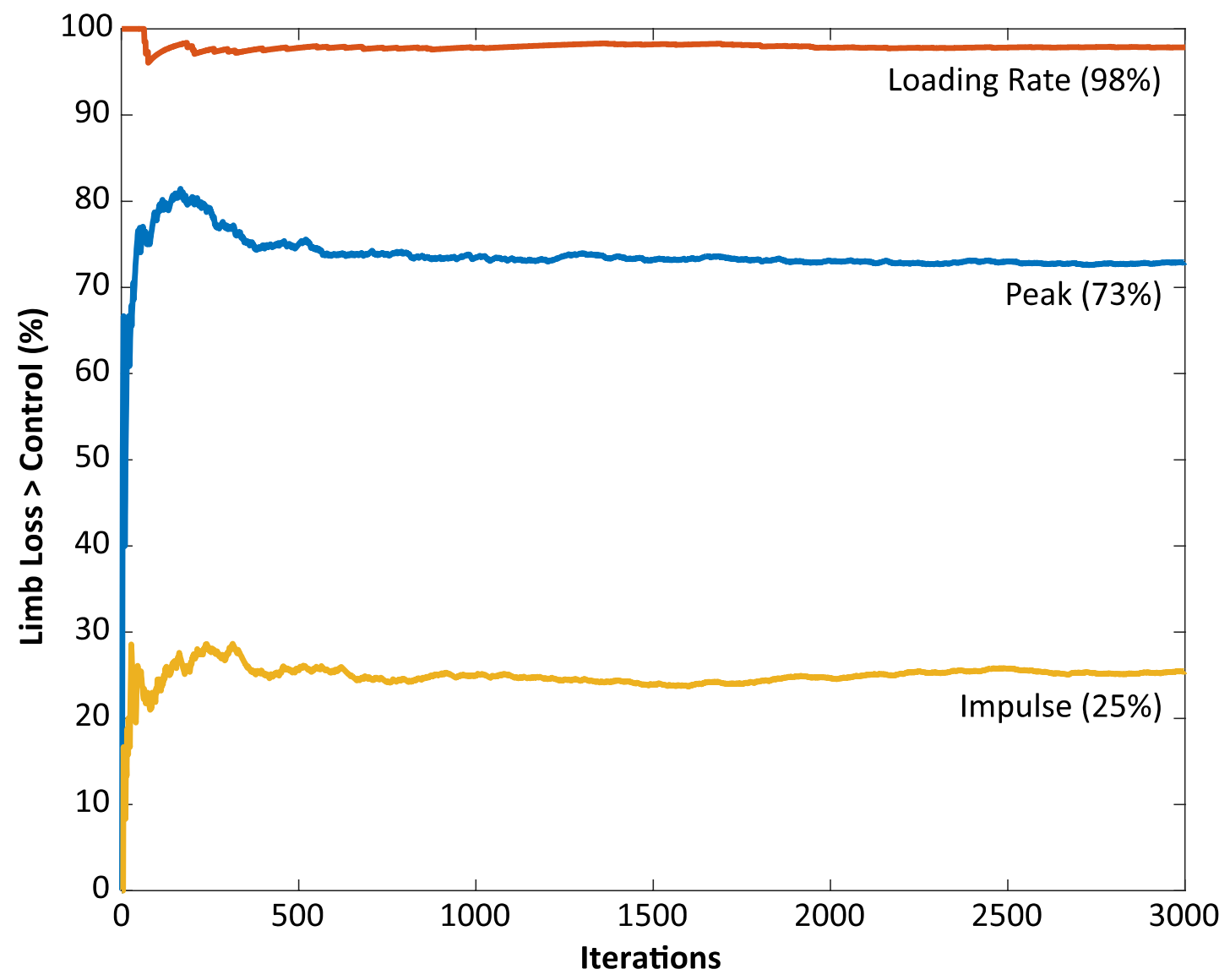

Figure 5. Monte Carlo simulation results for knee model parameter perturbations. The vertical axis shows the fraction of iterations for which the medial joint contact force outcome variable 588 was significantly greater in the limb loss group vs. the control group. The results using the 589 original (unperturbed) parameters are not included here. 

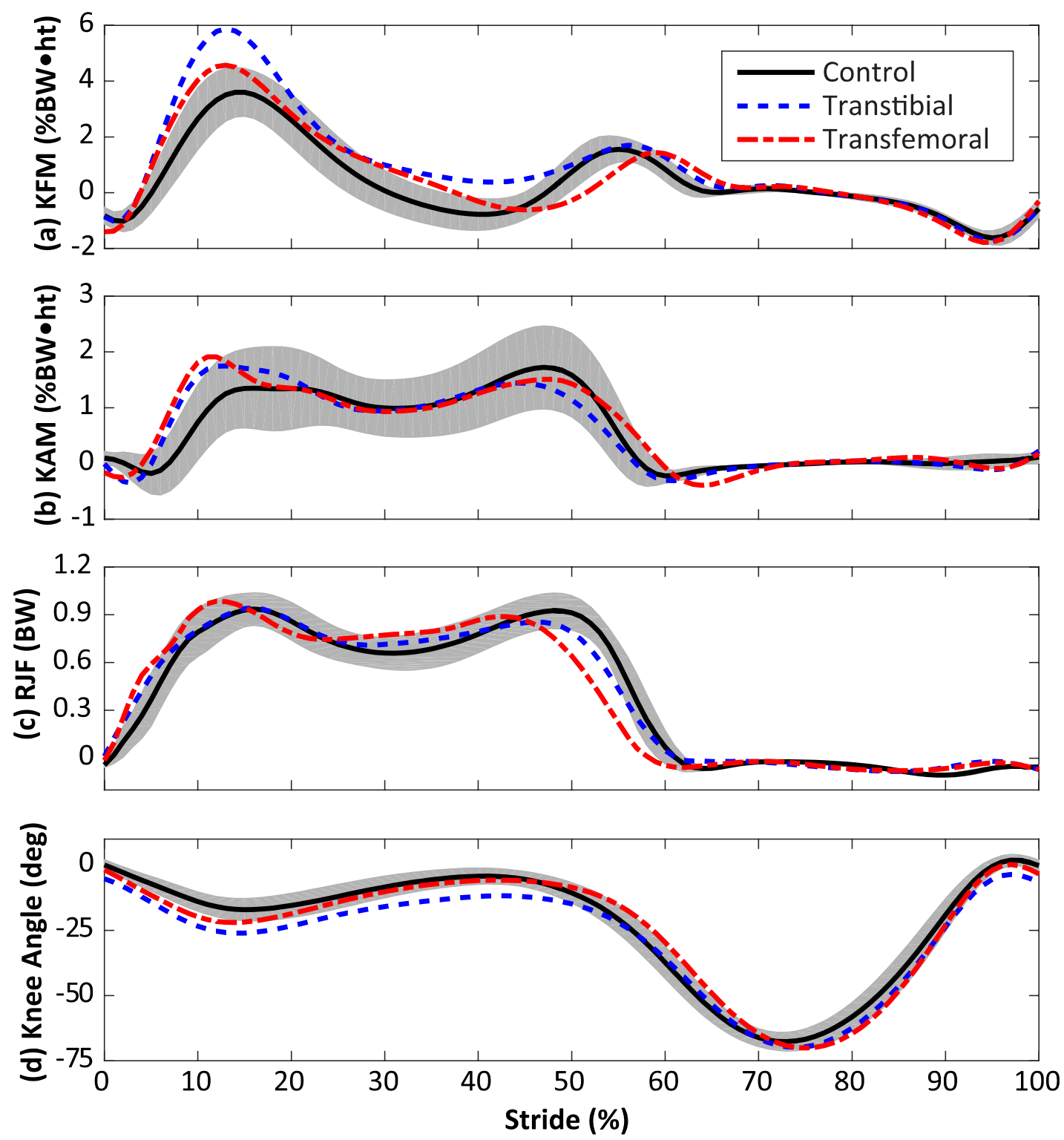

591 Figure 6. Knee flexion moment (KFM, a), knee adduction moment (KAM, b), resultant joint 592 force along the long axis of the shank (RJF, c), and knee flexion angle (d) during the stride, 593 beginning at heel-strike. Solid, dashed, and dash-dotted lines are means for control, transtibial, 594 and transfemoral subjects. The shaded areas are \pm one between-subjects standard deviation for 595 the control subjects. Scaling factors were bodyweight (BW) and height (ht). 
596 Table 1. Medial joint contact force model parameters. PCSA is physiological cross-sectional 597 areas. The three values shown for each moment arm and each muscle angle are values at $(0,-30$, $598-60)$ degrees of knee flexion, respectively, with 0 degrees defined as full extension. Muscle 599 angles are clockwise from the tibial plateau (anterior-positive and lateral-positive). Moment 600 arms and muscle angles were defined as second-order polynomials fit to these data.

\begin{tabular}{|c|c|c|}
\hline PCSA $\left(\mathrm{cm}^{2}\right)$ & Value & Arnold et al. (2010) \\
\hline Biceps femoris & 16.8 & \\
\hline Semimembranosus & 19.1 & \\
\hline Semitendinosus & 4.9 & \\
\hline Lateral gastrocnemius & 9.9 & \\
\hline Medial gastrocnemius & 21.4 & \\
\hline Sagittal moment arms (mm) & & Wretenberg et al. (1996) \\
\hline Biceps femoris & $(-21.5,-22.9,-24.4)$ & \\
\hline Semimembranosus & $(-35.6,-37.6,-41.3)$ & \\
\hline Semitendinosus & $(-25.6,-26.4,-31.2)$ & \\
\hline Lateral gastrocnemius & $(-38.7,-41.0,-47.0)$ & \\
\hline Medial gastrocnemius & $(-37.9,-40.4,-47.6)$ & \\
\hline Patellar tendon & $(50.8,50.6,44.1)$ & \\
\hline Frontal moment arms (mm) & & Wretenberg et al. (1996) \\
\hline Biceps femoris & $(48.8,48.4,48.6)$ & \\
\hline Semimembranosus & $(-33.5,-33.7,-30.1)$ & \\
\hline Semitendinosus & $(-29.7,-30.5,-26.3)$ & \\
\hline Lateral gastrocnemius & $(19.4,18.4,19.2)$ & \\
\hline Medial gastrocnemius & $(-8.6,-15.1,-17.1)$ & \\
\hline Patellar tendon & $(4.7,6.7,9.1)$ & \\
\hline Sagittal muscle angles (deg) & & Wretenberg et al. (1996) \\
\hline Biceps femoris & $(89.6,88.9,89.3)$ & \\
\hline Semimembranosus & $(107.5,100.3,98.7)$ & \\
\hline Semitendinosus & $(105.6,96.9,96.6)$ & \\
\hline Lateral gastrocnemius & $(73.7,65.6,61.3)$ & \\
\hline Medial gastrocnemius & $(74.0,67.9,64.3)$ & \\
\hline Patellar tendon & $(63.8,58.5,56.2)$ & \\
\hline Frontal muscle angles (deg) & & Wretenberg et al. (1996) \\
\hline Biceps femoris & $(102.0,100.6,100.9)$ & \\
\hline Semimembranosus & $(84.2,83.8,85.3)$ & \\
\hline Semitendinosus & $(84.7,89.1,89.4)$ & \\
\hline Lateral gastrocnemius & $(85.7,84.7,86.1)$ & \\
\hline Medial gastrocnemius & $(83.9,82.0,78.8)$ & \\
\hline Patellar tendon & $(101.3,98.0,95.7)$ & \\
\hline Distance b/w femoral condyles (cm) & 5.0 & Terzidis et al. (2012) \\
\hline
\end{tabular}

